\title{
Obtención de celulosa a partir de residuos de piña (Ananas comosus L. Merril)
}

\author{
Presenda-Gerónimo, Andrea ${ }^{1}$; Bolio-López, Gloria I. ${ }^{*}$; Veleva Lucien²; Azamar-Barrios, José Antonio ${ }^{2}$; Ramírez-Casillas, Rogelio ${ }^{3}$; \\ Sarracino-Martínez, Omar'; Hernández-Villegas, Manuel Mateo ${ }^{1}$; De la Cruz-Burelo, Patricia ${ }^{1}$ \\ ${ }^{1}$ Universidad Popular de la Chontalpa. Dirección Académica de Ciencias Básicas e Ingenierías; \\ ${ }^{2}$ Centro de Investigación y de Estudios Avanzados-Instituto Politécnico Nacional- Unidad Mérida; \\ ${ }^{3}$ Universidad de Guadalajara, Departamento de Madera Celulosa y Papel. \\ *Autor de correspondencia: gloria.bolio@upch.mx
}

\begin{abstract}
Objective: To obtain high quality cellulose from pineapple (Ananas comosus L. Merril) residues.

Design/methodology/approach: Pineapple crowns were used to obtain the cellulose using the $0.5 \%$ acid pre-hydrolysis treatment with sulfuric acid combined with the Sosa-Anthraquinone pulping method (SQ) and elemental chlorine-free bleaching (ECF). The cellulose obtained was characterized by the TAPPI techniques of Fourier Transformed Infrared Spectroscopy (FTIR), X-Ray Diffraction (XRD) and Scanning Electron Microscopy with coupled elemental analyzer (SEMEDX).

Results: It was demonstrated that cellulose-free cellulose was obtained from the chlorination and bleaching stages determined by SEM elemental analysis (EDX) with an alpha $(\alpha)$ cellulose content of 93\%, which exhibits the typical crystalline structure of type I cellulose commonly found in the natural plant fibers

Limitations of the study/implications: In the process, a relatively low fiber yield of $29.3 \%$ was obtained.

Findings/conclusions: Obtaining cellulose from pineapple residues (crowns) is proposed as an alternative of use due to its reinforcement capacity in composite materials, as well as in the production of biomaterials; showing that agricultural residues from pineapple are feasible to obtain cellulose.
\end{abstract}

Keywords: Soda-Antraquinone, Physicochemical characterization.

\section{RESUMEN}

Objetivo: obtener celulosa de alta calidad a partir de residuos de piña (Ananas comosus L. Merril).

Diseño/metodología/aproximación: Se utilizaron coronas de piña para la obtención de la celulosa utilizando el tratamiento de pre-hidrólisis ácida al 0.5\% con ácido sulfúrico combinado con el método de pulpeo Sosa-Antraquinona (SQ) y blanqueo libre de cloro elemental (ECF). La celulosa obtenida fue caracterizada por las técnicas TAPPI de Espectroscopia Infrarroja por Transformada de Fourier (FTIR), Difracción de Rayos-X (XRD) y Microscopía Electrónica de Barrido con analizador elemental acoplado (SEM-EDX).

Resultados: Se demostró obtener celulosa libre de residuos de las etapas de cloración y blanqueo determinado mediante el análisis elemental SEM (EDX) con un contenido de alfa $(\alpha)$ celulosa de $93 \%$, que exhibe la estructura cristalina típica de celulosa tipo I encontrada comúnmente en las fibras vegetales naturales. Limitaciones/implicaciones: En el proceso se obtuvo un rendimiento de fibra del $29.3 \%$ relativamente bajo.

Agroproductividad: Vol. 13, Núm. 2, febrero. 2020. pp: 77-82. Recibido: agosto, 2019. Aceptado: enero, 2020. 
Hallazgos/conclusiones: La obtención de celulosa a partir de los residuos de piña (coronas), se propone como una alternativa de uso debido a su capacidad de refuerzo en los materiales compuestos, así como en la elaboración de biomateriales; mostrando que los residuos agrícolas procedentes de la piña son factibles para la obtención de celulosa.

Palabras clave: Sosa-antraquinona, caracterización fisicoquímica.

\section{INTRODUCCIÓN}

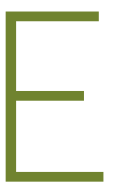

n la actualidad el sector agroindustrial, agrícola y doméstico generan grandes cantidades de residuos y anualmente cerca de 76 millones de toneladas son residuos orgánicos de frutas (Gonzáles lizado para su consumo en fresco y procesado, generando residuos que son fuente de contaminación, y con ayuda de los avances tecnológicos se busca un uso alternativo de este material (Espinoza et al., 2011). Estudios previos han demostrado la factibilidad de obtener material lignocelulósico de residuos agrícolas con métodos de pulpeo alcalino $(\mathrm{NaOH})$ para obtención de celulosa. La celulosa es una molécula lineal con moléculas de anillos de glucosa $\left(\mathrm{C}_{6} \mathrm{H}_{10} \mathrm{O}_{5}\right)$ ligada al $\mathrm{C} 1$ de un anillo de glucosa y al C4 del anillo adyacente a través de un enlace covalente de oxígeno (Azizi et al., 2005). En Tabasco, México, la producción anual de piña ocupa una extensión de 1,293.75 hectáreas anuales, produciendo 47,210 t (SNITT, 2016), de las cuales 20\% son residuos de coronas de piña (TICSO, 2014; Espinoza et al., 2011; Cano, 2008) potencialmente disponibles para el proceso de obtención de celulosa generando un uso alternativo de estos residuos. Por ello, el objetivo fue la obtención de celulosa a partir de esta fuente.

\section{MATERIALES Y MÉTODOS}

Se emplearon residuos de piña (Ananas comosus L. Merril), procedentes de las inmediaciones de Tierra Colorada 4ta. Sección, localizadas $17^{\circ} \mathrm{N}$ y $93^{\circ} \mathrm{W}$ del poblado C-34 del municipio de Huimanguillo, Tabasco, México. Los residuos fueron secados al sol, desmedulados en un refinador de discos Marca Sprout-Waldron posteriormente el material fibroso obtenido fue sometido a un tratamiento de prehidrólisis ácida suave con $0.5 \%$ ácido sulfúrico $\left(\mathrm{H}_{2} \mathrm{SO}_{4}\right)$ por una hora para la remoción de la lignina, hidromódulo 8 a 1, temperatura $130{ }^{\circ} \mathrm{C}$, presión de vapor 40 PSI y tiempo de reacción a temperatura máxima de 60 min. El tratamiento de prehidrólisis se llevó a cabo en un cocedor desfibrador, y después de la cocción, se lavó y centrifugó la fibra para ser puesta a secar a temperatura ambiente.

La obtención de la celulosa se realizó con el método de pulpeo Sosa-Antraquinona (SQ). En el pulpeo $S Q$, las condiciones fueron: carga de reactivo 15\%, concentración de antraquinona $0.1 \%$, hidromódulo de 10 a 1, temperatura máxima de $170{ }^{\circ} \mathrm{C}$ y tiempo de reacción a temperatura máxima de $60 \mathrm{~min}$. La fibra fue cocida en una solución de sosa cáustica y antraquinona como catalizador para la extracción de la mayor cantidad de lignina posible. Las pulpa fue sometida a cuatro etapas de blanqueamiento libre de cloro elemental
(ECF): (1) a la pulpa se le aplicó dióxido de cloro a una concentración del $10 \%$ con un pH inicial de 3 a 4 y se sometió a baño maría con la finalidad de eliminar lignina residual; (2) se añadió $\mathrm{NaOH}$ en una concentración del $10 \%$ con un pH inicial de 12 a 12.5 y se introdujo a baño maría para la solubilizar las hemicelulosas presentes en la pulpa y mejorar el blanqueamiento; (3) se aplicó a la pulpa dióxido de cloro al 2.5\% diluido a una concentración del 10\%, con un pH inicial de 5 a 5.5 y fue sometida a un baño maría ayudando al blanqueamiento con una reacción de oxidación. Posterior a esta etapa se realizó un pretratamiento con DTPA con el fin de atrapar los metales arrastrados en el proceso; (4) se utilizó Peróxido al 2\%, Sosa $(\mathrm{NaOH})$ $1 \%$, Sulfato de Magnesio $0.1 \%$ y Silicato de Sodio $0.2 \%$ a una concentración del 10\% con un pH inicial de 11.5 y se introdujo en un baño maría. Después de finalizar cada etapa se realizó el lavado de la pulpa con agua desionizada hasta obtener un $\mathrm{pH}$ neutro. Posteriormente a este tratamiento se obtuvo celulosa de alta calidad. Las fibras de celulosa se molieron en un molino MF 10 IKA WERKE, y se tamizaron en una malla de $0.5 \mathrm{~mm}$.

La caracterización fisicoquímica de la celulosa se llevó a cabo bajo las siguientes técnicas:

\section{Blancura, Deslignificación en Pulpa y Contenido de Alfa, Beta y Gama celulosa} Se aplicó la técnica TAPPI T 452 OM 92 para blancura en pulpa, papel y cartón (Reflectancia direccional a $457 \mathrm{~nm}$ ) con el equipo Spectrum Elrepho 3000, Técnica TAPPI T 236 CM 85 aplicada para la determinación de blanqueabilidad o grado de deslignificación de pulpa y la 
Técnica TAPPI T 203 OM 88 para determinar el contenido de alfa, beta y gamma celulosa.

\section{Espectroscopia de Infrarrojo}

Para determinar los grupos funcionales presentes en la celulosa se utilizó un Espectrofotómetro de Infrarrojo con Transformada de Fourier (FTIR) de la marca Perkin Elmer, modelo Spectrum GX con aditamento de reflectancia total atenuada (ATR) con cristal de diamantes y usando intervalos de medición de 4000 a $700 \mathrm{~cm}^{-1}$ y 16 barridos.

\section{Difracción de Rayos X (XRD)}

Mediante el uso de Difracción de Rayos X (XRD) métodos de polvos se obtuvieron los difractogramas de las muestras de celulosa. El índice de cristalinidad se calculó de acuerdo con el método de Segal (Segal, 1959) Ec. (1):

$$
\mathrm{Crl}=\left(/_{002}-I_{\text {amorph }}\right) / /_{002} \times 100 \%
$$

Donde /002 es el máximo de la intensidad de difracción (el plano 002) y la lamorph es la intensidad de difracción mínima (entre 101 y 002 picos). El tamaño del cristal fue calculado usando la ecuación de Scherrer Cullity (1978) Ec. (2):

$$
t=0.9 \lambda / B \cos \theta
$$

Donde $t$ es el tamaño del cristal, 0.9 es el factor de forma de cristal, $\lambda$ es la longitud de onda de la radiación utilizada $\left(\lambda_{C u}\right)$, $B$ el ancho a la mitad del pico de difracción de la muestra y el $\theta$ la posición del pico de difracción.

\section{Microscopía Electrónica de Barrido (SEM)}

Se analizó la morfología y diámetros de las fibras de celulosa mediante Microscopía Electrónica de Barrido con analizador elemental acoplado (SEM-EDX) Marca Bruker D8 Advance.

\section{RESULTADOS Y DISCUSIÓN}

En el proceso se obtuvo un rendimiento de fibra del 29.3\%. Mediante la técnica 1991 TAPPI "Determination of useful fiber in bagasse" el rendimiento promedio de fibra útil fue del $(15.07 \pm 0.3 \%)$ similares a $(11 \pm 4.0 \%)$ reportados por Ross, (2014) de celulosa obtenida a partir de las coronas de piña.
El Cuadro 1 muestra el rendimiento de pulpeo (\%) y No. Kappa técnica TAPPI 236 CM 85 (grado de deslignificación); el rendimiento del pulpeo SQ fue 55.3\%, el cual es menor al reportado por Bolio (2011) para raquis y pseudotallo de Musa cavendish con $66 \%$ y al reportado por Morán (2008) para fibra de sisal con 50 a 74\% de celulosa, y mayor al rendimiento en raquis del banano reportado por Restrepo, (2007) (51\%), de bagazo de caña de azúcar sin almacenar (54.3\%) y para el bagazo industrial seco (53\%) (García, 2013). Las diferencias se pueden atribuir a que fueron sometidos a diferentes concentraciones de cargas reactivas en los procesos de pulpeo. Khalil et al. (2011) menciona que los métodos que se emplean al obtener material celulósico dependen de la celulosa del material que se ocupa, su pretratamiento y que depende de su proceso de desintegración.

El Cuadro 1 muestra que el No. de Kappa para pulpa no blanqueada fue de $15.09 \%$ y en la pulpa blanqueada el No. de Kappa fue 5\%. Aguilar-Rivera (2010), reportó valores mayores para No. de Kappa de 18.1\% (bagazo sin almacenar) y $11.8 \%$ (bagazo industrial fresco). Los resultados muestran la manera eficiente de eliminación de lignina debido a que los valores encontrados están dentro de los estándares con No Kappa (10-15) para pulpa no blanqueada con el método empleado. Mediante el estudio en el SPECTRUM Elrepho 3000 Serie datacolor No. 1700. Se determinó la blancura, brillantez y los colores CIE L*.a.*b de la hoja blanqueada con blanqueo libre de cloro elemental (ECF) de acuerdo a la técnica TAPPI T 452 OM 92 para blancura en pulpa, papel y cartón (Reflectancia direccional a $457 \mathrm{~nm}$ ) mostrando los resultados obtenidos en el Cuadro 2, donde la blancura promedio fue de 89.4\%, la cual, es similar a los reportados por García (2013), para el bagazo de caña de azúcar con valores (88.8 $\pm 0.2-89.9 \pm 0.2 \%)$ obtenida con $\mathrm{NaOH}$ y blanqueo dióxido, extracción, dióxido (DED) y ligeramente menor que el bagazo sin almacenar con 89.9\% y mayor al bagazo industrial fresco con $81.2 \%$ reportados por Aguilar-Rivera (2010), utilizando pulpeo a la sosa y blanqueo convencional a base de compuestos clorados mediante la secuencia cloración en medio ácido, extracción alcalina y blanqueo con hipoclorito alcalino (CEH).

$\begin{aligned} & \text { Cuadro 1. Propiedades físicas de las hojas de corona } \\
& \text { de piña. }\end{aligned}$
\begin{tabular}{l|r} 
Propiedad / pulpa & $\begin{array}{l}\text { Pulpeo sosa } \\
\text { antraquinona }\end{array}$ \\
\hline Rendimiento en pulpeo (\%) & 55.30 \\
\hline No. Kappa pulpa no blanqueada & 15.09 \\
\hline No. Kappa pulpa blanqueada & 5.00 \\
\hline
\end{tabular}

Se puede deducir que el método de pulpeo y blanqueo utilizado en el estudio es eficiente, debido, a que los valores de blancura son mayores a $81.2 \%$ 
Cuadro 2. Valores de blancura de la hoja Sosa-antraquinona. Luminosidad ( $\left.L^{\star}\right)$, Rojo - verde ( $\left.a^{\star}\right)$, Amarillo - Azul ( $\left.b^{\star}\right)$.

\begin{tabular}{c|c|c|c|c|c}
\hline$L^{*}$ & $a^{*}$ & $b^{*}$ & $440 \mathrm{~nm}$ & $460 \mathrm{~nm}$ & Desviación Estándar \\
\hline 96.56 & -0.67 & 2.07 & 87.54 & 89.22 & \pm 0.18 \\
\hline 95.75 & -0.56 & 1.55 & 88.02 & 89.57 & \pm 0.18 \\
\hline 96.25 & -0.67 & 2.05 & 88.68 & 90.02 & \pm 0.18 \\
\hline 96.01 & -0.59 & 1.69 & 88.50 & 89.8 & \pm 0.18 \\
\hline
\end{tabular}

y ligeramente menor a $89.9 \%$ aunque el valor óptimo se encuentra entre el $90 \%$ y más.

En el año 2004, Navarro mencionó que la hoja blanqueada superficialmente encontrada en un sector $b^{*}$ positivo, es decir con coloración amarillo que se hace menos amarillo para valores de máxima blancura y también en el sector $a^{*}$ negativo con coloración verde es una desventaja, ya que se prefiere un blanco óptico de color azul por razones estéticas. El Cuadro 3 muestra los resultados del contenido de alfa $(\alpha)$, beta $(\beta)$ y gamma $(\gamma)$ celulosa en la pulpa obtenida por el método de pulpeo $S Q$, donde el resultado promedio de $(\alpha)$ celulosa fue de $93 \%$, $(\beta)$ celulosa de $5.75 \%$ y $1.25 \%$ de $(\gamma)$ celulosa, de acuerdo a García et al. (2013), los valores reportados para el bagazo de caña de azúcar fueron ligeramente similares con $(91.2 \pm 0.3-$ $93.5 \pm 0.3 \%)$ de $(\alpha)$ celulosa y aproximadamente un $7.7 \%$ pertenece a $(\beta)$ y $(\gamma)$ celulosa.

Lo anterior sugiere que las hojas de corona de piña son fuente para la obtención de celulosa de acuerdo al contenido de $(\alpha)$ celulosa obtenido.

La Figura 1 muestra el Interferograma de la celulosa obtenida por el método SQ. La banda de grupos $\mathrm{O}-\mathrm{H}$ a $3300 \mathrm{~cm}^{-1}$ es más ancha y aparece al analizar muestras sólidas que contienen agua y puede comprobarse debido a que existe una banda entre $1630 \mathrm{~cm}^{-1}$ y $1650 \mathrm{~cm}^{-1}$ (contreras et al., 2010) y el pico a $2900 \mathrm{~cm}^{-1}$ se asigna a vibraciones de estiramiento de C-H (Lu y Hsieh, 2010) y según Qazanfarzadeh (2016) son característicos de estiramientos de $\mathrm{C}-\mathrm{H}$ y flexión de los grupos $\mathrm{CH}_{2}$. En la banda a $1640 \mathrm{~cm}^{-1}$ se observa la flexión correspondiente a los grupos $\mathrm{O}-\mathrm{H}$ pertenecientes al agua absorbida por la fibra y la banda a 1370 $\mathrm{cm}^{-1}$ es característico de la celulosa (Morán, 2008).

La intensidad a la banda $1160 \mathrm{~cm}^{-1}$ es atribuida a enlaces C-O-C; la bandas a $1109 \mathrm{~cm}^{-1}$ presenta flexiones en el plano, y los estiramientos C-O caen en la misma región (Lui et al., 2017); mientras los picos en $1054 \mathrm{~cm}^{-1}$ y $1030 \mathrm{~cm}^{-1}$ son carac- terísticos del enlace C-O (Contreras et al., 2010). Ross (2014), menciona que el pico $1050 \mathrm{~cm}^{-1}$ es asignado

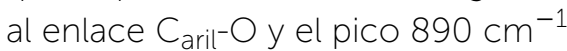
corresponde a hidrógenos aromáticos, confirmando así la remoción de lignina y hemicelulosa.

La Figura 2 muestra los difractogramas de la celulosa obtenida de corona de piña $S Q$, con picos cercanos a $2 \theta=15.8^{\circ} 23^{\circ}$ y $35^{\circ}$. De acuerdo con Wang y Wen (2017), los picos observados cercanos a $2 \theta=14.9^{\circ}, 16.7^{\circ}, 20.6^{\circ}, 22.8^{\circ}$ y $34.4^{\circ}$ exhiben la estructura de la celulosa de tipo I, para los planos de difracción 101, 101, 021, 002 y 004 encontrada comúnmente en las fibras vegetales naturales, así como los

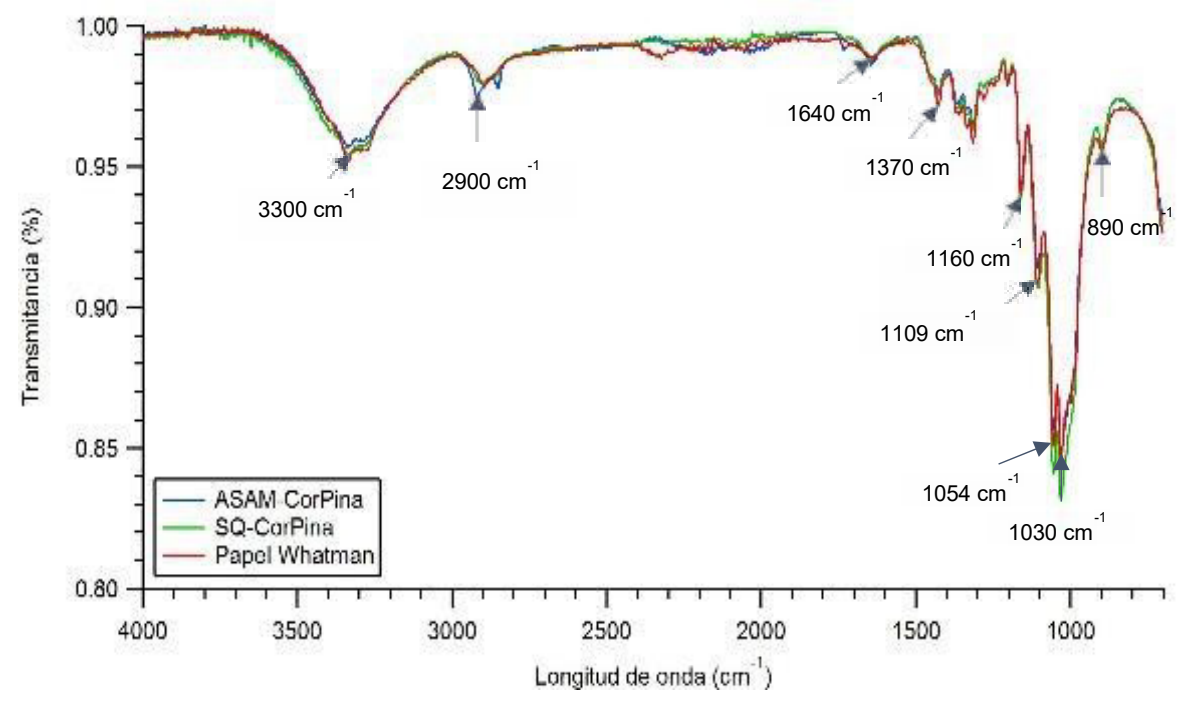

Figura 1. Interferograma de muestras de celulosa corona de piña método SQ.

Cuadro 3. Contenido de Alfa $(\alpha)$, Beta $(\beta)$ y Gamma $(\gamma)$ Celulosa.

Proceso

Sosa-antraquinona \%
Alfa-celulosa

93

Beta-celulosa
Gama-celulosa 
picos $2 \theta=15.4^{\circ}, 16.2^{\circ}$ y $34.6^{\circ}$ reportados por ( Visakh, 2010) y Ross (2014) reportó picos cercanos a $2 \theta=12.6^{\circ}, 20.6^{\circ}, 21.8^{\circ}$ y $34.4^{\circ}$

La cristalinidad para la celulosa fue de $71.4 \%$ con un tamaño de cristal de 63.65 nm (63.35 A), comparado con Ross (2014) quien reportó una menor cristalinidad (43.05 \pm 1.5$)$ con un tamaño de cristal de 2.30 nm (2.30 A) a partir de hojas de corona de piña y Bolio et al., (2011), reportaron un valor similar (72\%) para celulosa extraída a partir del raquis y pseudotallo de Musa cavendish y un tamaño de cristal menor 4.46 nm (4.46 A). Comprobando de esta manera que el tamaño de los cristales puede aumentar o disminuir por efecto de factores que alteran la cristalinidad, de acuerdo a la fuente de origen de la celulosa, métodos de su extracción y tratamientos posteriores a su extracción (Bolio et al., 2016).

La micrografía electrónica de barrido Figura 3 (a) muestra los diámetros de celulosa (1.81 $\mu \mathrm{m}, 2.06 \mu \mathrm{m}$ y $3.12 \mu \mathrm{m}$ ), obteniendo un diámetro promedio $(2.33 \pm 0.78 \mu \mathrm{m}) \mathrm{me}$ nor al diámetro promedio de $(2.9025 \pm 0.75 \mu \mathrm{m})$ reportado por Ross (2014).
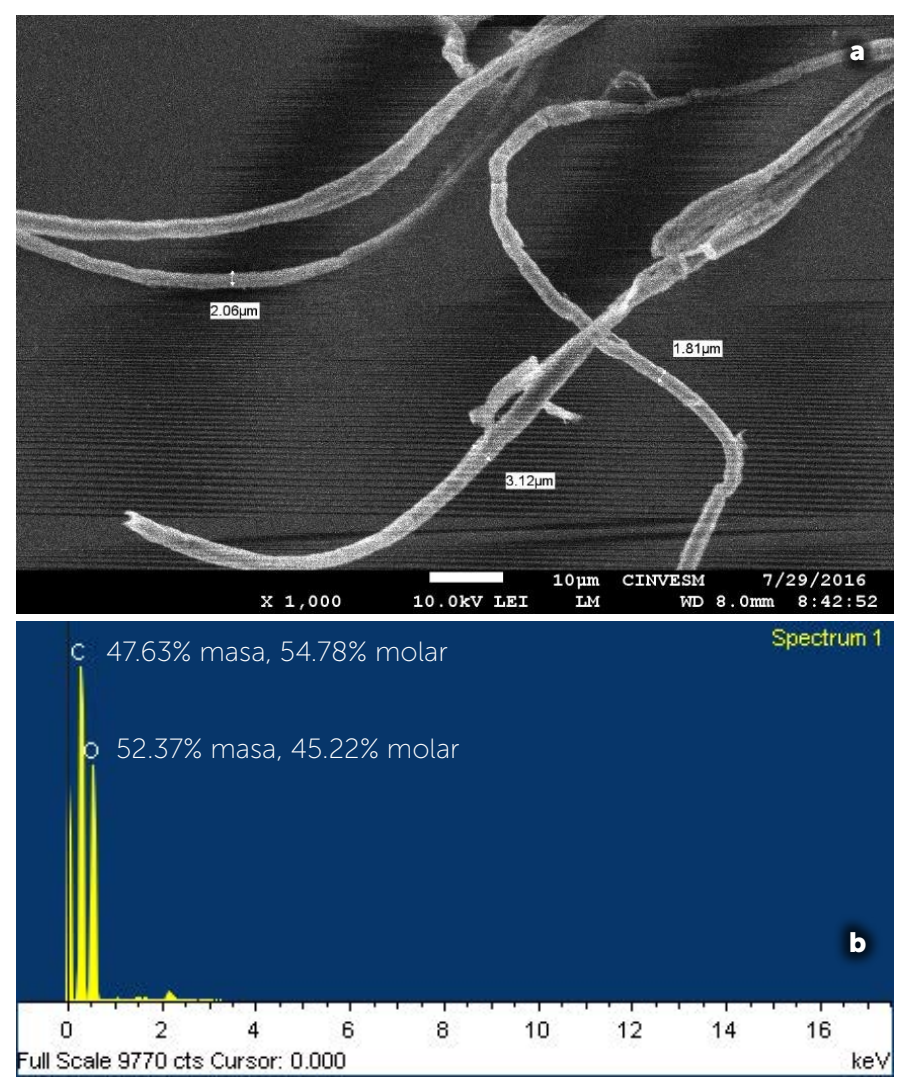

Figura 3. (a) Micrografía SEM de celulosa de corona de piña SQ, (b) Análisis elemental SEM celulosa corona de piña SQ.

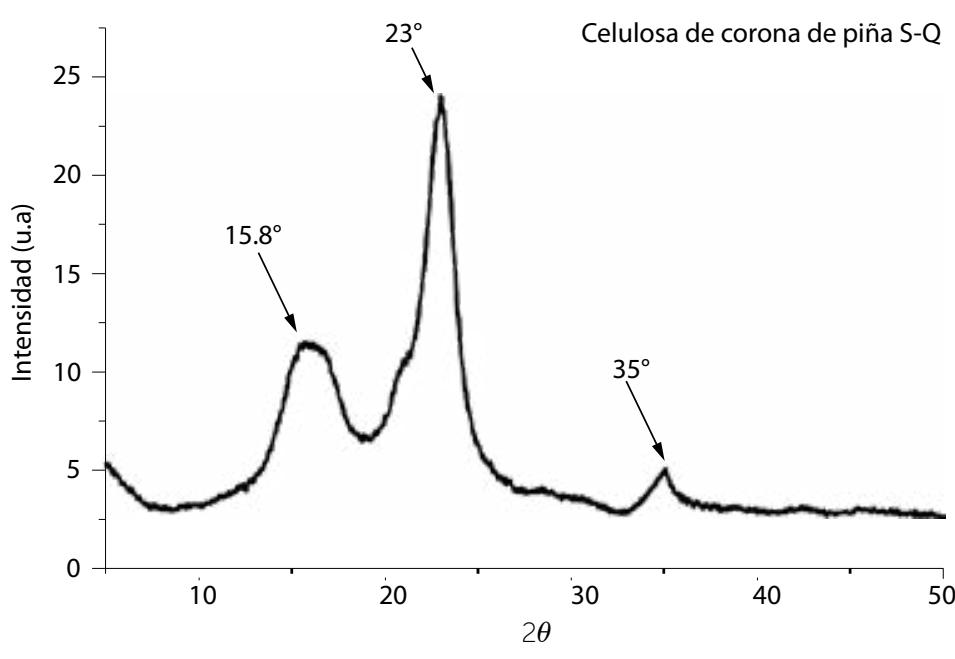

Figura 2. Difractograma celulosa coronas de piña $S Q$.

En el análisis elemental (EDX), Figura 3 (b), se observa como los principales elementos constituyentes de la celulosa, carbono y oxígeno: 1. Carbono (C) con $47.63 \%$ masa y $54.78 \%$ molar valores menores a $54.18 \%$ masa y $61.5 \%$ molar según Ross (2014), y 2. Oxígeno (O) con una composición de $52.37 \%$ masa y $45.22 \%$ molar mayores a $44.68 \%$ masa y $38.09 \%$ molar. Con este estudio se comprueba que no existen residuos de las etapas de cloración y blanqueo

\section{CONCLUSIONES}

El tratamiento de pre-hidrólisis ácida al 0.5\% con ácido sulfúrico combinado con el método de pulpeo SQ y blanqueo libre de cloro elemental (ECF), demostró obtener celulosa libre de residuos de las etapas de cloración y blanqueo determinado mediante el análisis elemental SEM (EDX) con un contenido de alfa $(\alpha)$ celulosa de $93 \%$, que exhibe la estructura cristalina típica de celulosa tipo I encontrada comúnmente en las fibras vegetales naturales y se propone como alternativa de uso debido a su capacidad de refuerzo en los materiales compuestos, asi como en la elaboración de biomateriales

\section{AGRADECIMIENTOS}

Los autores agradecen el financiamiento de este trabajo mediante el proyecto integral "APROVECHAMIENTO SUSTENTABLE DEL CULTIVO DE PIÑA (Ananas comosus L. Merril) EN MÉXICO" Clave DSA/103.5/15/11123 de la Red de Investigación e Innovación en Sustentabilidad Agroindustrial (RIISA). Programa de Desarrollo Profesional Docente para el tipo Superior (PRODEP)-SEP.

\section{LITERATURA CITADA}

Aguilar-Rivera, N. (2010). Efecto del almacenamiento de bagazo de caña en las propiedades físicas de celulosa grado papel. Rev. 
Ingeniería Investigación y Tecnología. 13: 189-197. ISSN 14057743 FI-UNAM.

Azizi Samir, M. A. S., Alloin F., Paillet M., \& Dufresne A. (2004). Tangling Effect in Fibrillated Cellulose Reinforced Nanocomposites. Macromolecules. 37: 4313- 4316.

Bolio, G., Valadez, A., Veleva, L. y Andreeva, A. (2011). Whiskers de Celulosa a partir de Residuos Agroindustriales de banano: Obtención y Caracteriación. Rev. Mex. Quím. 10 (2): 291-299. ISSN 1665-2738.

Bolio, G., Veleva, L., Hernández, V. M. y Salgado, G. S. (2016) Obtención de Celulosa a partir de Bagazo de Caña de Azúcar (Saccharum spp.), Agroproductividad: Vol. 9 Núm. 7. Pp: 41-45

Cano, A. H. (2008). Diagnóstico del Manejo Postcosecha, Comercialización e Industrialización de Piña (Ananas comosus L. Merril) en Huimanguillo, Tabasco, México. (Tesis de Maestro en Ciencias) Colegio de Posgraduados Campus Tabasco, H. Cárdenas, Tabasco. Pp. 2-24.

Contreras Q., J. H., Trujillo, P., Hugo, A., Arias, O., Pérez, C., José, L., Delgado, F. (2010). Espectroscopía ATR-FTIR de Celulosa: Aspecto Instrumental y Tratamiento Matemático de Espectros. e- Gnosis. 8: 1-13. E-ISSN: 1665- 5745H.

Cullity, B. D. (1978). Elements of X-Ray Diffraction Second Edition Addison Wesley, New York.

Espinoza, N., Cadena, P., De la Cruz F. R., y González I. (2011). El Aguacate una Especie muy Mexicana. Caracterización Social y Técnica del Cultivo de la Piña Criolla (Ananas comosus L. Merril). Biofertilizantes a Base de Micorriza- arbuscular y su Aplicación en la Agricultura y El Proceso de Fermentación del Cacao. En: AGRO productividad. 1: 4.

García, L., Bordallo, E., Dopico, D. y Cordero, D. (2013). Obtención de Celulosa Microcristalina a partir del Bagazo de la Caña de Azúcar. Rev. ICIDCA. 47: 57-63. ISSN 0138-6204.

González M. y Pérez S. et al., (2015). Residuos Agroindustriales con Potencial para la Producción de Metano Mediante la Digestión Anaerobia. Rev Argent Microbiol. 47(3). Pp. 230.

Khalil, H.P.S., Bhat, A. H., y Yusra, A.F. (2011). Green Composites Sustainable Cellulose Nanofibrils: A review. En: Carbohydrate Polymers. 87: 963-979.

Lu, P. y Hsieh, Y. L. (2010). Preparation and Properties of Cellulose Nanocrystals: Rod, Spheres, and Network. Carbohydrate Polymers. 82: 329-336.
Lui, J., Holuszko, M., Mastalerz, M. (2017). Applications of micro-FTIR Technique in Studying Hydrophobicity of Coal. International Journal of Coal Geology. COGEL 2826.

Morán, J. (2008). Extracción de Celulosa y Obtención de Nanocelulosa a partir de Fibra Sisal. 2do. Encuentro de Jóvenes Investigadores en Ciencia y Tecnología de Materiales. Argentina. Pp. 1-6.

Qazanfarzadeh Zeinab. (2016). Properties of Whey Protein Isolate Nanocomposite Films Reinforced with Nanocellulose Isolated From Oat Husk. S0141-8130 (16)30623-7.

Restrepo, A., Zuluaga, R., Castro, C., Correa, N., Vélez, J., y Gañán, P. (2007). Nano-composites Reforzados con Microfibrillas de Celulosa Aisladas de Paredes Celulares del Raquis de Banano. Rev. Scientia et Technica Año XIII. 36: 689-693. ISSN 01221701.

Ross, A., R.E. (2014). Obtención y Caracterización de Celulosa a Partir del Residuo Agroindustrial de Piña (Ananas comosus L. Merril). División de Ciencias Básicas e Ingenierías, México: Universidad Popular de la Chontalpa. Tesis de Licenciatura. Pp. 33-36.

Segal, L., Creely, J., Martin, A., Conrad, C. (1959). An empirical method for estimating the degree of crystallinity of native cellulose using the X-ray diffractometer. Text. Res. J. 29(10): 786-794.

SNITT, 2016. Agenda Nacional de Investigación, Innovación y Recursos Fitogenéticos 2016-2022.

Técnica Technical Association of the Pulp and Paper Industry - TAPPI Blancura en Pulpa, Papel y Cartón 452 OM 92.

Técnica Technical Association of the Pulp and Paper Industry - TAPPI No. KAPPA o grado de deslignificación 236 CM 85

Técnica Technical Association of the Pulp and Paper Industry - TAPPI T Contenido de Alfa, Beta y Gamma celulosa 203 OM 88.

Ticso, (2014). Aprovechamiento Residuos Biomasa de Producción de Piña (Ananas comosus) para Municipio de Aguazul Casanare. Tecnologías de la Información y las Comunicaciones de la Orinoquia.1: 2 .

Visakh, P. M. y Thomas, S. (2010). Preparation of Bionanomaterials and their Polymer Nanocomposites from Waste and Biomass. Waste Biomass Valor 1: 121-134.

Wang H., Wen X., Wei Y., Wang X. y Liu C. (2017). Preparation and Characterization of Cellulose Laurate Ester by Catalized Transesterification. Carbohydrate Polymers. 168: 247-254. 